CERN-TH/95-202

BARI-TH/211-95

\title{
The Higgs Boson Mass from Precision Electroweak Data
}

\author{
John Ellis ${ }^{a}$, G.L. Fogli ${ }^{b}$ and E. Lisi ${ }^{b, c}$ \\ a Theory Division, CERN, CH-1211, Geneva, Switzerland \\ ${ }^{b}$ Dipartimento di Fisica and Sezione INFN di Bari, Bari, Italy \\ ${ }^{c}$ Institute for Advanced Study, Princeton, NJ 08540
}

\begin{abstract}
We present a new global fit to precision electroweak data, including new low- and high-energy data and analyzing the radiative corrections arising from the minimal symmetry breaking sectors of the Standard Model (SM) and its supersymmetric extension (MSSM). It is shown that present data favor a Higgs mass of $\mathcal{O}\left(M_{Z}\right)$ :

$$
M_{H}=76 \stackrel{+152}{-50} \mathrm{GeV} .
$$

We confront our analysis with (meta)stability and perturbative bounds on the SM Higgs mass, and the theoretical upper bound on the MSSM Higgs mass. Present data do not discriminate significantly between the SM and MSSM Higgs mass ranges. We comment in passing on the sensitivity of the Higgs mass determination to the values of $\alpha\left(M_{Z}\right)$ and $\alpha_{s}\left(M_{Z}\right)$.
\end{abstract}

CERN-TH/95-202

BARI-TH/211-95

July 1995 


\section{Introduction}

The discovery of the top quark by the CDF [1] and D0 [2] collaborations with a mass that agrees to within $10 \%$ with that predicted from precision electroweak data [3, 4, 5] constitutes an impressive success for the Standard Model, confirming its predictivity at the level of quantum loops.

The fact that the Standard Model (SM) is renormalizable [6] if and only if the top quark and the Higgs boson are included implies that loop corrections are sensitive to the masses $\left(m_{t}, M_{H}\right)$ of these particles, with the sensitivity to $m_{t}$ being much stronger than that to $M_{H}$ [7].

Low-energy data and early measurements of $M_{W}$ were used to constrain $m_{t}$ before the start of SLC and LEP physics [8], at which time the sensitivities of $Z$ decay observables to $m_{t}$ and $M_{H}$ were well known [9]. It was pointed out that these calculations could be used to predict $m_{t}$ once a precise measurement of $M_{Z}$ became available [10]. Subsequently, the use of this and other precision $Z$ decay observables to predict $m_{t}$ has become a major focus of interest in LEP and SLC physics [1], 3, [, 5].

For some time now, the precision electroweak data have also exhibited some sensitivity to $M_{H}$ [12, 13, and the success of the $m_{t}$ prediction is now shifting the focus of interest to the prediction of $M_{H}$ [3, औ, 14], particularly in view of the advent

of LEP 2 physics and the drive to optimize the continuation of the Higgs search at the LHC. The precision electroweak data have consistently favored $M_{H}<300$ $\mathrm{GeV}$, and it is important when considering the maximum energy of LEP 2 and the low-mass Higgs search at the LHC to understand how seriously to take this trend.

This understanding is also relevant for indications on the direction of particle physics beyond the Standard Model. The (meta)stability of the electroweak vacuum [15, 16] imposes a lower limit on $M_{H}$ that depends on $m_{t}$ and the scale $\Lambda_{V}$ up to which the Standard Model effective potential is assumed to represent physics accurately. There is also an upper limit on $M_{H}$ that follows from requiring the Standard Model couplings to remain perturbative up to a scale $\Lambda_{P}$ [17]. On the other hand, in the Minimal Supersymmetric extension of the Standard Model (MSSM), constraints on the form of the effective potential impose an intrinsic upper limit 
on the lightest Higgs mass of order $150 \mathrm{GeV}$. It is thus important to see how the indirect determinations of the Higgs mass in the SM and the MSSM compare with the above limits, the composite (technicolor) Higgs option being in serious conflict with electroweak data 18.

The main purpose of this paper is to discuss $M_{H}$ in the light of the recent direct determination of $m_{t}$ and the latest round of precision electroweak data from LEP and elsewhere. We argue that the combined direct and indirect data now favour significantly $M_{H}<300 \mathrm{GeV}$. We confront the indirect determination of $M_{H}$ with theoretical bounds from vacuum stability and supersymmetry. We conclude that all the experimental results and theoretical bounds are consistent with both the SM and the MSSM. We attempt to quantify the relative probabilities of the Higgs mass ranges in the SM and MSSM, finding an indication that the MSSM may be preferred. This indication is not yet significant, but may become so in the future. In passing, we supplement our discussion of the electroweak precision data analysis with a more technical issue: the impact of $\alpha\left(M_{Z}\right)$, in the light of various recent re-evaluations [19, 20, 21, 22], showing in particular how future measurements of the muon $g_{\mu}-2$ [23] can improve our knowledge of $\alpha\left(M_{Z}\right)$.

\section{Data Analysis and Fits to $m_{t}$}

Our procedure for fitting the available electroweak data is basically the same as we have described in previous works [3, 11, 12], so here we just comment on the new data that have recently become available, and the way we treat them.

Foremost are the measurements of $m_{t}$ by CDF [1]: $m_{t}=176 \pm 8$ (stat) \pm 10 (sys) $\mathrm{GeV}$, which we interpret as $176 \pm 13 \mathrm{GeV}$, and by D0 [2]: $m_{t}=199_{-21}^{+19}$ (stat) \pm 22 (sys) $\mathrm{GeV}$, which we interpret as $199 \pm 29 \mathrm{GeV}$. In 1994, the compatibility of the indications from CDF [24] and the absence of an indication from D0 [25] was an issue, as was compatibility with theoretical calculations of the $t \bar{t}$ production cross-section [26]. These are no longer issues, as the CDF and D0 measurements of $m_{t}$ are highly compatible, allowing us to combine them to obtain $m_{t}=181 \pm 12 \mathrm{GeV}$, and the cross-section measurements are now also highly compatible with each other and quite consistent with the theoretical calculations. 
In addition, the LEP electroweak working group has made available a new set of precision electroweak measurements [4] based on increased statistics of over $1.1 \times 10^{7}$ $Z$ decays. The most significant improvements have been a $50 \%$ reduction in the error on the $Z$ mass measurement, $M_{Z}=91.1887 \pm 0.0022 \mathrm{GeV}$, and a $30 \%$ reduction in the error in the hadronic cross-section measurement $\sigma_{h}^{0}=41.492 \pm 0.081 \mathrm{nb}$. There have also been significant reductions in the errors on $\Gamma_{Z}, A_{F B}^{\ell}, A_{\tau}$ and $A_{e}$. Also important is a shift by more than one standard deviation in the central value of $A_{F B}^{b}$ to $0.1015 \pm 0.0036$, which brings it into significantly better agreement with the global electroweak fit parameters. On the other hand, the apparent discrepancy in $R_{b}$ has not been reduced. In our analysis we assume that this apparent discrepancy is not due to new physics.

Concerning the SLD measurement of $A_{L R}$ (or, equivalently, of $\sin ^{2} \theta_{\text {eff }}^{\text {lept }}$ ), the latest value is [27]: $A_{L R}=0.1551 \pm 0.0040\left(\sin ^{2} \theta_{\text {eff }}^{\text {lept }}=0.2305 \pm 0.0005\right)$, corresponding to the global 1991-95 SLD data sample. This should be compared to the 1991-93 SLD value [28]: $A_{L R}=0.1656 \pm 0.0076\left(\sin ^{2} \theta_{\text {eff }}^{\text {lept }}=0.2294 \pm 0.0010\right)$, and to the LEP value [4: $\sin ^{2} \theta_{\text {eff }}^{\text {lept }}=0.2320 \pm 0.0004$. It is evident that, as far as $\sin ^{2} \theta_{\text {eff }}^{\text {lept }}$ is concerned, the latest SLD central value is now closer to the LEP value, although the reduction of the SLD error means that the values are still about $2 \sigma$ apart. Nevertheless, we include $A_{L R}$ in our global fit.

Other new elements in our fit are an updated value for the $W$ mass: $M_{W}=$ $80.33 \pm 0.17 \mathrm{GeV}$ (world average) [29], and two new measurements of parity violation in atomic Thallium that have recently been reported: $\mathcal{R}=\operatorname{Im}\left\{E 1^{P N C} / M 1\right\}=$ $(-15.68 \pm 0.45) \times 10^{-8}$ [30] and $(-14.68 \pm 0.17) \times 10^{-8}$ [31]. The power of these two Thallium experiments in constraining electroweak radiative corrections is comparable to that of atomic Cesium results [32]. Apart from the inclusion of the above new atomic result, our treatment of the available low-energy precision electroweak data is identical with that documented in our previous works [3, 11, 12]. We emphasize that treating the deep-inelastic $\nu N$ scattering cross-section measurements as measurements of $1-M_{W}^{2} / M_{Z}^{2}$ (see, e.g., Ref. 画) is only an approximation, and that there are other significant low-energy electroweak measurements that we include in the global fit 33 .

Figure 1 shows the results of global fits within the Standard Model to the available electroweak measurements, as contours of $\Delta \chi^{2}=1,4$ in the $\left(M_{H}, m_{t}\right)$ plane. 
We recall that their projections onto the coordinate axes correspond to $\pm 1 \sigma$ and $\pm 2 \sigma$ errors on the top and Higgs masses. The dashed lines are fits that do not include the combined CDF and D0 measurements of $m_{t}$, which is shown as an error bar on the left. Projecting the $\Delta \chi^{2}=1$ dashed ellipse on the vertical axis, we find

$$
m_{t}=156_{-15}^{+14} \mathrm{GeV}
$$

for the Standard Model fit to the precision electroweak data with $M_{H}$ left free, with a minimum $\chi_{\min }^{2}=12.2$. Our central value of $m_{t}$ in (1) is somewhat lower than that quoted by the LEP electroweak working group [4], mainly because we do not fix the central value of $M_{H}$ at $300 \mathrm{GeV}$, and partly as a result of our more complete treatment of the available low-energy data (that prefer a relatively "light" top). Fig. 2 shows the contributions of the various different electroweak sectors to the $\chi^{2}$ function of the global fit for the particular choice $M_{H}=M_{Z}$, and Fig. 3 shows the global $\chi^{2}$ functions for a sampling of different values of $M_{H}$. We have checked that, if we restrict our fit to the LEP data alone, and assume the same value of $M_{H}(300$ $\mathrm{GeV}$ ), our central value of $m_{t}$ agrees with theirs within $3 \mathrm{GeV}$, which is within the typical theoretical uncertainties.

The small size of the error in (1) is a tribute to the precision of the LEP experiments, in particular. The range in (1) is compatible with the CDF/D0 measurements, although somewhat lower. This compatibility is an impressive confirmation of the Standard Model at the one-loop level, and justifies combining the direct and indirect information on $m_{t}$. The solid lines in Fig. 1 are the $\Delta \chi^{2}=1,4$ contours for such a combined fit, whose projection on the vertical axis yields

$$
m_{t}=172 \pm 10 \quad \mathrm{GeV}
$$

The $\chi_{\min }^{2}$ of the global fit is increased by $\Delta \chi^{2}=1.8$ to $\chi_{\min }^{2}=14.0$ when the CDF and D0 measurements of $m_{t}$ are included. This increase in $\chi^{2}$ is acceptable $(<1.4 \sigma)$, and the total $\chi^{2} /$ d.o.f. remains of order unity. 


\section{Implications for $M_{H}$}

We now turn to the discussion of $M_{H}$, which is the main purpose of this paper. Projecting the $\Delta \chi^{2}=1$ contours of Fig. 1 on the horizontal axis, we find for the fit to the precision electroweak data alone

$$
M_{H}=36 \begin{aligned}
& +56 \\
& -22
\end{aligned} \quad \mathrm{GeV} \quad\left[\log _{10}\left(M_{H} / M_{Z}\right)=-0.40 \begin{array}{l}
+0.40 \\
\end{array}\right]
$$

and for the fit that includes also the CDF/D0 $m_{t}$ measurement:

$$
M_{H}=76 \begin{aligned}
& +152 \\
& -50
\end{aligned} \quad \mathrm{GeV} \quad\left[\begin{array}{ll}
\log _{10}\left(M_{H} / M_{Z}\right)=-0.08 & +0.48 \\
& -0.46
\end{array}\right]
$$

In each case, we have restated the fit result in a logarithmic scale, since the leading dependences of the experimental observables on $M_{H}$ are logarithmic. We note that the errors are fairly symmetric in this scale, reflecting the fact that the $\chi^{2}$ function is well-behaved in $\log _{10}\left(M_{H} / M_{Z}\right)$ around the absolute minimum. This is seen in Fig. 4, which displays $\chi^{2}$ as a function of $M_{H}$ (on a logarithmic scale) for a sampling of choices of $m_{t}$. The envelope of these $\chi^{2}$ functions is the $\chi^{2}$ function for $M_{H}$ with $m_{t}$ free, corresponding to the projection of Fig. 1 on the horizontal axis.

We have verified that the shape of the $\chi^{2}$ function we find is similar to that found by the LEP electroweak working group [4] if we restrict our fit to a similar data set. It is clear that the default value $M_{H}=300 \mathrm{GeV}$ assumed by the LEP electroweak working group in quoting central values of $m_{t}$ is not the most probable value, and is indeed more than $1 \sigma$ away far from it. We stress again that, because of the well-known positive correlation between $m_{t}$ and $M_{H}$ visible in Fig. 1, this assumed value of $M_{H}$ is the main reason the LEP electroweak working group quotes a higher central value of $m_{t}$ than we do in (1) and (2).

We have also verified that the shape of the $\chi^{2}$ function found by Swartz [34] in a

fit using a very similar data set is similar to ours, though obtained with a different treatment of the low-energy data. 


\section{Variations in the Analysis}

Before discussing the predicted range [eqs. (3), (4)] of $M_{H}$ in more detail, we comment on how our analysis is affected by uncertainties in $\alpha_{s}\left(M_{Z}\right)$ and by the $A_{L R}$ measurement at SLD. Then we consider in more detail the impact of $\alpha\left(M_{Z}\right)$. In Fig. $1, \alpha_{s}\left(M_{Z}\right)$ is fixed at the best fit value, 0.124 (to which we attach an uncertainty of \pm 0.005$)$. This is somewhat higher than the world average: $\alpha_{s}\left(M_{Z}\right)=0.117 \pm 0.007$ 35]. Imposing $\alpha_{s}\left(M_{Z}\right)=0.117$ in the fit, the value of $\chi_{\min }^{2}$ increases by $\sim 1.8$, but $M_{H}$ diminishes by only $\sim 7 \mathrm{GeV}$, and $m_{t}$ is not significantly affected. We conclude that the uncertainty in $\alpha_{s}\left(M_{Z}\right)$ is not an important factor at present in the analysis of $M_{H}$.

Concerning $A_{L R}$, it is well-known that the SLD value tends to bring $M_{H}$ down with respect to the rest of the electroweak data [3]. However, even excluding $A_{L R}$ completely, a procedure that we do not consider justified, we find that the central values of $\log _{10}\left(M_{H} / M_{Z}\right)$ in eqs. (3) and (4) are increased by about +0.16 and +0.28 respectively, namely less than the corresponding $1 \sigma$ uncertainty in $\log _{10}\left(M_{H} / M_{Z}\right)$.

We now turn to the sensitivity of our results to the assumed value of $\alpha\left(M_{Z}\right)$. In the past, we have taken $\alpha\left(M_{Z}\right)^{-1}=128.87 \pm 0.12$ from Ref. [36], but recently there have been several re-evaluations of the extrapolation from the Thompson limit, some of which differ appreciably from the earlier value [36]. In this paper we have assumed $\alpha\left(M_{Z}\right)^{-1}=128.896 \pm 0.090$ from Ref. [19], which is similar to the recent estimate in Ref. [2] (128.89 \pm 0.090$)$. We now explore the implications of varying $\alpha\left(M_{Z}\right)^{-1}$ within the range suggested by other estimates [20, 22] f. Fig. 5 shows the values of a subset of electroweak observables $\left(\sin ^{2} \theta_{e f f}^{l e p}, M_{W}\right.$ and $\left.\Gamma_{Z}\right)$ in the $m_{t}$ range indicated by CDF and D0 and for three choices of $M_{H}$ (this is not a fit). The left-hand side of the figure is for $\alpha\left(M_{Z}\right)^{-1}=128.896 \pm 0.090$, and the right-hand side for a value $2 \sigma$ higher, namely $\alpha\left(M_{Z}\right)^{-1}=129.076 \pm 0.090$, similar to the evaluation of 22. The minor axes of the theoretical ellipses in Fig. 5 are due to the propagation of the error in $\alpha\left(M_{Z}\right)^{-1}$. We see that the effects on $M_{W}$ and $\Gamma_{Z}$ of varying $\alpha\left(M_{Z}\right)$ are very small, and that the effect on $\sin ^{2} \theta_{\text {eff }}^{\text {lep }}$ is to bring the theoretical predictions closer to the

1 The central value in Ref. [20] (128.99 \pm 0.06$)$ and Ref. 22] (129.08 \pm 0.10$)$ are about 1 and $2 \sigma$ above the central values in Refs. [19, 21]. We have recently been informed [M. Swartz, private communication] that an update of Ref. [22] yields a value close to Refs. [19]. 
SLD measurement. However, it is evident in the same Fig. 5 that even this $2 \sigma$ shift in the electromagnetic coupling constant is less relevant in the theory/experiment comparison than the dispersion of the LEP/SLD data, and thus it does not affect significantly the stability of the $M_{H}$ range discussed previously.

Conversely, we can ask if future precision electroweak data can improve our knowledge of $\alpha\left(M_{Z}\right)$. A $2 \sigma$ variation in $\alpha\left(M_{Z}\right)^{-1}$ can induce a few $\mathrm{GeV}$ shift in $m_{t}$ at fixed $M_{H}$ (see, e.g., Ref. [22]), so it is not impossible that the combination of future, more precise direct (CDF/D0) and indirect (LEP/SLD) determinations of $m_{t}$ with an error of $\sim 5 \mathrm{GeV}$ could also reduce implicitly the uncertainty in $\alpha\left(M_{Z}\right)^{-1}$.

Such future improvements may also be linked to future more precise $g_{\mu}-2$ measurements possible with the BNL E821 experiment [23]. The reason is that the theoretical determinations of the hadronic contribution to $\alpha\left(M_{Z}\right)^{-1}$ and $g_{\mu}-2$ are correlated, since the same set of $e^{+} e^{-} \rightarrow$ hadrons data is used in their dispersion integral estimates, although with different convolution kernels. Assuming full correlation of the partial systematic errors induced in $\alpha\left(M_{Z}\right)$ and $g_{\mu}-2$ by the different independent low-energy subsets of the data compiled in Ref. [19], we have estimated the theoretical joint standard deviation ellipse in the $\left[\alpha\left(M_{Z}\right), g_{\mu}-2\right]$ plane (Fig. 6). Also shown in Fig. 6 is the situation (dotted ellipse) to be expected after prospective improvements in measuring hadron production at DA $\Phi$ NE and VEPP-2M (see [19 and references therein), where we see that the correlation between $\alpha_{e m}\left(M_{Z}\right)^{-1}$ and $g_{\mu}-2$ becomes stronger. Also shown is the prospective error in $g_{\mu}-2$ expected to be obtained by the BNL E821 experiment (horizontal band). We see that, by virtue of this correlation, the anticipated measurement in this experiment could even serve to constrain the possibile range of $\alpha\left(M_{Z}\right)$. The vertical band reminds us the possibility of fitting a value of $\alpha\left(M_{Z}\right)$ from future precision data, as is now done with $\alpha_{s}\left(M_{Z}\right)$, though its width is purely hypothetical.

\section{Implications of Our Analysis of $M_{H}$}

In view of the remarkable stability of the $M_{H}$ range in Fig. 1, the indication for a relatively light Higgs mass of $\mathcal{O}\left(M_{Z}\right)$ should be taken seriously. The upper limit at $2 \sigma\left(M_{H} \lesssim 700 \mathrm{GeV}\right.$, including $\left.\mathrm{CDF} / \mathrm{D} 0\right)$ is reassuringly below the $\mathrm{TeV}$ region, so 
the perturbative calculations which the fit is based upon are expected to be reliable. The upper end of the $1 \sigma$ range $\left(M_{H} \lesssim 230 \mathrm{GeV}\right)$ and the central value $M_{H}=76$ $\mathrm{GeV}$ give hope for finding the Higgs at the LEP2 or the LHC. In general, it is definitely non-trivial that the electroweak data consistently favour a Higgs mass in a range of $\mathcal{O}\left(M_{Z}\right)$, which disfavours composite or strongly-interacting scenaria, as discussed elsewhere 18 .

The question arises whether this range is compatible with bounds on the SM Higgs mass derived from (meta)stability of the electroweak vacuum, and from perturbative behaviour of the SM couplings. In the upper part of Fig. 7 we plot first the same $\Delta \chi^{2}=1$ contour as in Fig. 1 (CDF/D0 included), the dashed part representing the LEP direct limit $M_{H}>65 \mathrm{GeV}$. Superposed are the lower limits on $M_{H}$ from vacuum metastability requirements [16], as a function of the "new physics" scale $\Lambda_{V}$ in $\mathrm{GeV}$ up to which the effective potential in the SM is assumed to apply (bounds from absolute stability of the SM vacuum [15] would be weaker by a few $\mathrm{GeV}$ for our central value of $m_{t}=172 \mathrm{GeV}$ ). The $M_{H}$ range we find is compatible with the (meta)stability bounds, particularly if $\Lambda_{V}$ is small, but it is not yet possible to exclude any value of $\Lambda_{V}$ and thus give any indication on the possible new physics scale. Also shown in Fig. 7 are upper bounds on $M_{H}$ obtained by requiring the SM couplings to remain perturbative up to a scale $\Lambda_{P}$. We see that these are also compatible with our analysis, particularly if $\Lambda_{P}$ is small, though again we cannot exclude any range of this scale. In the particular case $\Lambda_{V}=\Lambda_{P}=10^{19} \mathrm{GeV}$ and $m_{t}=172 \mathrm{GeV}$ (our central value), these bounds become $116 \mathrm{GeV}<M_{H}<190$ $\mathrm{GeV}$.

In the minimal supersymmetric extension of the Standard Model (MSSM) the Higgs sector depends on the pseudoscalar Higgs mass $M_{A}$, the v.e.v. ratio $v_{2} / v_{1}=$ $\tan \beta$ and the value of the top mass, through radiative corrections to the Higgs potential. We assume fixed, large values for the other MSSM parameters, so that the remaining MSSM spectrum decouples. Then, for any given value of $\tan \beta$, the radiative corrections induced by the MSSM Higgs sector are specified by the lightest Higgs mass $m_{h}$ and $m_{t}$, which are the coordinates of the lower plot in Fig. 7 . The previous metastability bounds do not apply to the MSSM vacuum. However, new intrinsic upper bounds on $m_{h}$ appear, as shown for two representative values of $\tan \beta$ $(\tan \beta=2,16)$. For $m_{t}=172 \mathrm{GeV}$ (our central value), the upper limit on $m_{h}$ in the 
MSSM is $124 \mathrm{GeV}$. For $m_{h} \sim \mathcal{O}\left(M_{Z}\right)$, the radiative corrections arising from the SM and MSSM Higgs sector differ only by small subleading terms, and the similarity of the $\chi^{2}$ functions in the SM and the MSSM has been demonstrated in previous analyses [3, 18], hence the similarity of the $\Delta \chi^{2}=1$ contours in the upper and lower halves of Fig. 7.

We conclude this paper by proposing an exploratory interpretation of our results addressed to a possible comparison between the SM and the MSSM. In Fig. 8 we show the cumulative probabity $P\left(M_{H}\right)$, calculated from the behaviour of the SM $\chi^{2}$ function shown in Fig. 4, integrated appropriately over $m_{t}$ and including the measurements from CDF [1] and D0 [2]. We note that this full cumulative probability distribution does not apply to the MSSM, because of the intrinsic upper limit on $m_{h}$ mentioned in the previous paragraph. However, we can use the cumulative probability curve in Fig. 8 to compare the SM and the MSSM by estimating the relative probabilities of the mass ranges allowed in the two models when other experimental and/or theoretical constraints, not incorporated in the structure of the electroweak radiative corrections, are taken into account. This comparison may be made using the SM curve in Fig. 8, because, as already mentioned, the $\chi^{2}$ functions for the SM and the MSSM are quite similar in the mass range around $M_{Z}$ which contains the bulk of the probability distribution [3].

In the case of the SM, we have a direct experimental lower limit $M_{H}>65 \mathrm{GeV}$ [4], but also the stronger metastability lower bound of $116 \mathrm{GeV}$ and the perturbative upper bound of $190 \mathrm{GeV}$ mentioned earlier. We estimate from Fig. 8 a total probability of $18 \%$ for the mass range $116 \mathrm{GeV}<M_{H}<190 \mathrm{GeV}$. In the case of the MSSM, the direct experimental lower bound on $m_{h}$ is somewhat weaker, and may be taken as $50 \mathrm{GeV}$, and there is no metastability lower bound, only the intrinsic upper bound of $124 \mathrm{GeV}$. We estimate from Fig. 8 a total probability of $36 \%$ for the mass range $50 \mathrm{GeV}<M_{H}<124 \mathrm{GeV}$. The relative probability is clearly higher for the MSSM than for the SM, but not significantly so.

The limitations and approximations inherent in this exploratory analysis are many and obvious. However, it provides us with a clear message: the data are surprisingly consistent with the MSSM, perhaps even more consistent than with the SM. 


\section{Acknowledgements}

The work of E.L. is supported in part by an I.N.F.N. post-doctoral fellowship, and by funds of the Institute of Advanced Study (Princeton). 


\section{References}

[1] CDF Collaboration, F. Abe et al., Phys. Rev. Lett. 74 (1995) 2676.

[2] D0 Collaboration, S. Abachi et al., Phys. Rev. Lett. 74 (1995) 2632.

[3] J. Ellis, G.L. Fogli and E. Lisi, Phys. Lett. B333 (1994) 118.

[4] The LEP Collaborations ALEPH, DELPHI, L3 and OPAL, and the LEP Electroweak Working Group, CERN Report No. LEPEWWG/95-01.

[5] G. Altarelli, R. Barbieri and F. Caravaglios, Nucl. Phys. B405 (1994) 3;

J. Erler and P. Langacker, Phys. Rev. D52 (1995) 441;

G. Montagna, O. Nicrosini, G. Passarino and F. Piccinini, Phys. Lett. B335 (1994) 484;

V.A. Novikov, L.B. Okun, A.N. Rozanov, M.I. Vysotskii and V.P. Yurov, Phys. Lett. B331 (1994) 433;

D. Schaile, Fortschr. Phys. 429 (1994) 429.

[6] G.'t Hooft, Nucl. Phys. B35 (1971) 167; ibidem B37 (1971) 195.

[7] M. Veltman, Nucl. Phys. B123 (1977) 89; Acta Phys. Polon. 8 (77) 475.

[8] U. Amaldi et al., Phys. Rev. D36 (1987) 1385;

G. Costa et al., Nucl. Phys. B297 (1988) 244;

J. Ellis and G.L. Fogli, Phys. Lett. B213 (1988) 526.

[9] G. Altarelli, R. Kleiss and C. Verzegnassi eds., Z Physics at LEP I, CERN Report No. 89-08 (Geneva, 1989) and references therein.

[10] J. Ellis and G.L. Fogli, Phys. Lett. B231 (1989) 189; ibidem B232 (1989) 139.

[11] J.Ellis, G.L. Fogli and E. Lisi, Phys. Lett. B292 (1992) 427.

[12] J.Ellis, G.L. Fogli and E. Lisi, Phys. Lett. B249 (1990) 543; ibidem B274 (1992) 456 .

[13] F. del Aguila, M. Martinez and M. Quirós, Nucl. Phys. B381 (1992) 451; V.A. Novikov, L.B. Okun, M.I. Vysotskii and V.P. Yurov, Phys. Lett. B308 (1993) 123. 
[14] P.H. Chankowski and S. Pokorski, Max-Planck Institute Report No. MPI$\mathrm{Ph} / 95-39$.

[15] G. Altarelli and I. Isidori, Phys. Lett. B337 (1994) 141;

J.A. Casas, J.R. Espinosa and M. Quirós, Phys. Lett. B342 (1995) 171.

[16] J.R. Espinosa and M. Quirós, Phys. Lett. B353 (1995) 257.

[17] M. Sher, Phys. Rep. 179 (1989) 274.

[18] J. Ellis, G.L. Fogli and E. Lisi, Phys. Lett. B343 (1995) 282.

[19] S. Eidelman and F. Jegerlehener, Paul Scherrer Institute (Villigen) Report No. PSI-PR-95-1.

[20] A.D. Martin and D. Zeppenfeld, Phys. Lett. B345 (1995) 558.

[21] H. Burkhardt and B. Pietrzyk, Lab. de Physique des Particules (Annecy) Report No. LAPP-EXP-95-05.

[22] M.L. Swartz, Report No. SLAC-PUB-6710 (revised version).

[23] B. Lee Roberts (BNL E821), Z. Phys. C56 (1992) S101.

[24] CDF Collaboration, F. Abe et al., Phys. Rev. D50 (1994) 2966; Phys. Rev. Lett. 73 (1994) 225.

[25] D0 Collaboration, S. Abachi et al., Phys. Rev. Lett. 72 (1994) 2138.

[26] E. Laenen, J. Smith and W. van Neerven, Phys. Lett. B321 (1994) 254 and references therein.

[27] SLD Collaboration, as presented at CERN by C. Baltay (June 1995)

[28] SLD Collaboration, K. Abe et al., Phys. Rev. Lett. 73 (1994) 25.

[29] CDF Collaboration, F. Abe at al., Report No. FERMILAB-PUB-95-033 (1995), to appear in Phys. Rev. D.

[30] N.H. Edwards et al., Phys. Rev. Lett. 74 (1995) 2654.

[31] P.A. Vetter et al., Phys. Rev. Lett. 74 (1995) 2658. 
[32] M.C. Noecker, B.P. Masterson and C.E. Wieman, Phys. Rev. Lett. 61 (1988) 310.

[33] CHARM-II Collaboration, P. Vilain et al., Phys. Lett. B281 (1992) 159.

[34] M. Swartz, private communication (1995).

[35] S. Bethke, Physikalisches Institut (Aachen) Report No. PITHA-94-30 (1994).

[36] F. Jegerlehner, in Testing the Standard Model, M. Cvetič and P. Langacker eds. (World Scientific, Singapore, 1991) p.476; Prog. Part. Nucl. Phys. 27 (1991) 32 . 


\section{Figure Captions}

Fig. 1 - Combined fit to all precision electroweak data in the $\left(M_{H}, m_{t}\right)$ plane, including (solid lines) or not (dashed lines) the direct determination of $m_{t}$ by CDF/D0 (error bar on the left). The contours correspond to $\Delta \chi^{2}=1,4$ around the minimum (small circle) in either case. Notice that $M_{H}$ is significantly below $300 \mathrm{GeV}$ at the $1 \sigma$ level, and below $1 \mathrm{TeV}$ at the $2 \sigma$ level.

Fig. 2 - The contributions to $\Delta \chi^{2}$ due to different sectors of the precision electroweak data set, as functions of $m_{t}$ for an assumed value $M_{H}=M_{Z}$.

Fig. 3 - The values of $\chi^{2}$ as functions of $m_{t}$ for the various indicated values of $M_{H}$.

Fig. 4 - The values of $\chi^{2}$ as functions of $M_{H}$ for the various indicated values of $m_{t}$.

Fig. 5 - The impact of a hypothetical shift of $\alpha\left(M_{Z}\right)^{-1}$ on selected electroweak observables $\left(\sin ^{2} \theta_{\text {eff }}^{\text {lept }}, M_{W}, \Gamma_{Z}\right)$. The three sub-figures on the left show the predictions (slanted ellipses) for such observables, assuming: the indicated value of $\alpha\left(M_{Z}\right)^{-1}$ (error included) [19], the CDF/D0 measurement of $m_{t}$, and three representative values of $M_{H}(65,300$ and $1000 \mathrm{GeV})$. The gray horizontal stripes represent the corresponding experimental determinations. If the central value of $\alpha\left(M_{Z}\right)^{-1}$ is increased by 2 standard deviations, the three sets of predictions on the right are obtained. Notice that the most significant effect is that on $\sin ^{2} \theta_{\text {eff }}^{\text {lept }}$.

Fig. 6 - One-standard-deviation ellipse corresponding to present theoretical estimates 19 of $\alpha\left(M_{Z}\right)^{-1}$ and $g_{\mu}-2$ (solid line). Also shown as a dotted ellipse is the envisaged reduction in the uncertainty that will come from future lowenergy experiments (mainly DA $\Phi \mathrm{NE}$ ). Notice the non-negligible correlation in both cases. The gray horizontal stripe represents a possible outcome of the high-precision $g_{\mu}-2$ experiment E821 at BNL [23]. The gray vertical stripe reminds us that some valuable indirect information on $\alpha\left(M_{Z}\right)^{-1}$ will be provided by the combination of more precise future electroweak measurements.

Fig. 7 - Comparison of combined top-Higgs mass fits in the Standard Model (SM, upper plot) and in its Minimal Supersymmetric extension (MSSM, lower plot), at $\Delta \chi^{2}=1$. The continuation of the $\Delta \chi^{2}=1$ contour below the LEP direct 
limit $M_{H}>65 \mathrm{GeV}$ is shown dashed. Also shown in the SM plot are the lower limits on $M_{H}$ from vacuum metastability [16] as a function of the "new physics" scale $\Lambda_{V}=10^{4}-10^{19} \mathrm{GeV}$, and the upper limts that come from requiring the SM couplings to remain perturbative up to a scale $\Lambda_{P}=10^{3}-10^{19} \mathrm{GeV}$. In the MSSM plot, the dashed region indicates $m_{h}$ below $50 \mathrm{GeV}$, and we show the intrinsic upper limits on the lightest Higgs mass for two values (2 and 16) of $\tan \beta=v_{2} / v_{1}$.

Fig. 8 - The cumulative probability distribution calculated from the $\chi^{2}$ function in the SM shown in Fig. 4, obtained after integrating appropriately over $m_{t}$, including the direct measurements from CDF [1] and D0 [2]. This may be used to estimate the relative probabilities of different Higgs mass ranges in the SM and the MSSM, as discussed in the text. 